\title{
Nasal glioma with psammomatous calcification- An unusual presentation
}

\author{
Rana K Sherwani ${ }^{1}$, Kafil Akhtar ${ }^{1 *}$, Prasenjit S Ray ${ }^{1}$, Sayed S Ahmad ${ }^{2}$ \\ ${ }^{I}$ Department of Pathology, Jawaharlal Nehru Medical College, Aligarh Muslim University, Aligarh, India. \\ ${ }^{2}$ Department of Oral Maxillo-facial Surgery, Sir Ziauddin Dental College, Aligarh Muslim University, Aligarh, India.
}

Received January 14, 2014; Revised March 18, 2014; Accepted March 20, 2014; Published Online April 09, 2014

\section{Case Report}

\begin{abstract}
Congenital midline swellings of nose are encountered rarely, and nasal gliomas constitute about 5\% of such lesions. Various theories have been suggested to explain the pathogenesis. Imaging preferably by MRI is mandated to study the extent and to rule out intracranial extension. Clinically, these masses are firm and incompressible. Histologically, they are made up of astrocytes and neuroglial cells, embedded in fibrous and vascular connective tissue. The mainstay of treatment is conservative surgical excision because nasal gliomas are slow-growing, rarely recurrent, and have no malignant potential. We present a case of congenital extranasal glioma with psammomatous calcification and without any intracranial extension in an eighteen month old boy.
\end{abstract}

Keywords: Extranasal Glioma; Child; Psammomatous Calcification

\section{Introduction}

The developmental anomalies of the nose encompass a diverse group of conditions, which include nasal dermoids, gliomas, encephaloceles, nasal clefts, proboscis lateralis, polyrrhinia, nasopharyngeal teratoma, and epignathus. ${ }^{1}$ The most common are nasal dermoids, nasal gliomas and nasal (meningo) encephaloceles, which are clinically significant because some of them might have an effective or potential connection with the central nervous system (CNS).

Nasal gliomas are one form of the congenital midline nasal masses that usually present at birth. They are rare, benign, congenital tumours, which arise from abnormal embryonic development. They are nonencapsulated CNS masses of neurogenic origin, which have lost their intracranial connections, and present as an obvious external or intranasal mass at birth, without associated surgical symptoms. ${ }^{2,3}$ The term "nasal glioma" is a confusing misnomer as it implies a neoplastic condition with malignant potential, which it is not. Around 15 to $20 \%$ of nasal gliomas have a fibrous stalk

*Corresponding author: Dr. Kafil Akhtar; Department of Pathology, Jawaharlal Nehru Medical College, Aligarh Muslim University, Aligarh, India.

Cite this article as:

Sherwani RK, Akhtar K, Ray PS, Ahmad SS. Nasal glioma with psammomatous calcification- An unusual presentation. Int J Cancer Ther Oncol2014; 2(2):02027. DOI: 10.14319/ijcto.0202.7 connecting to the central nervous system. It should be differentiated from glioma, which is a malignant tumor of the brain. In addition, it should be differentiated from a primary encephalocele, which is herniation of the cranial contents through a bone defect in the skull, through which it retains an intact connection with the central nervous system. ${ }^{4,5}$ An accurate diagnosis permits proper management and prevents potentially life-threatening intracranial complications. Severe complications such as meningitis or a brain abscess can be avoided if the lesions are removed at an early stage. We report a case of extranasal glioma with psammomatous calcifications in eighteen months old boy.

\section{Case Report}

An eighteen month old boy presented with a swelling over his nasal bridge. The swelling had been present since birth but did not cause any nasal obstruction. There was no history of epistaxis or any other nasal discharge. He was born at full-term and had a normal vaginal delivery. His elder sibling had no congenital abnormalities and the family history was unremarkable. On physical examination, the boy had a $3 \times 3$ $\mathrm{cm}$ superficial purple, soft to firm mass with a smooth surface over his nasal bridge. It was neither tender nor pulsatile. There was no intranasal mass. Clinically, the mass was diagnosed as nasal dermoid/hemangioma. 
Magnetic resonance imaging (MRI) of the brain showed a well-defined soft tissue attenuation lesion, at the root of the nose without any underlying bony defect or intracranial extension, measuring $2.9 \times 2.9 \times 2.5 \mathrm{~cm}$. (Figure 1)The lesion showed mild heterogenous enhancement with evidence of concentric calcifications within the lesion.

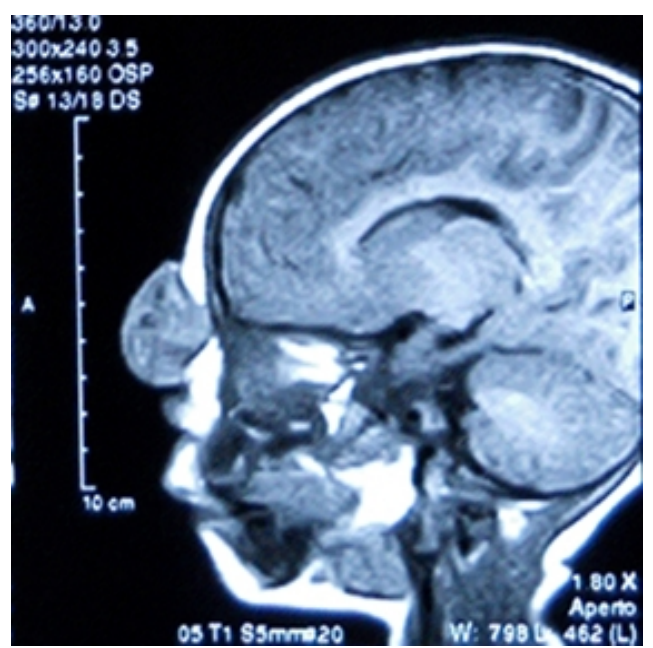

FIG. 1: MRI of the brain shows a well-defined soft tissue attenuation lesion, at the root of the nose without any underlying bony defect or intracranial extension, measuring $2.9 \times 2.9 \times 2.5 \mathrm{~cm}$.

The tumour was excised externally under general anaesthesia, using a left lateral elliptical skin incision. The margin was taken down to the cartilaginous pyramid. Grossly, it appeared as a lobulated, well-circumscribed, and unencapsulated whitish pink firm mass. Microscopic sections demonstrated the presence of glial tissue with a fibrillary background and interspersed fibrovascular stroma with psammomatous calcifications. (Figure 2 and 3). No meningeal or dural tissue was identified. The glial nature of the tumour was confirmed by a positive reaction for S100 protein (Figure 4 ), and a definitive diagnosis of nasal glioma was made.

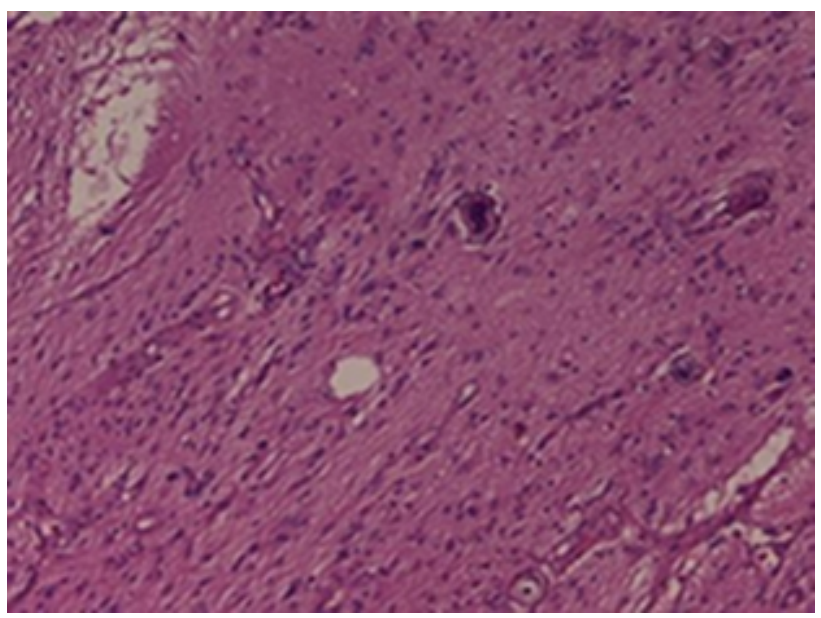

FIG. 2: Photomicrograph shows glial tissue with a fibrillary background and interspersed fibrovascular stroma. Haematoxylin and Eosin $\times 10$

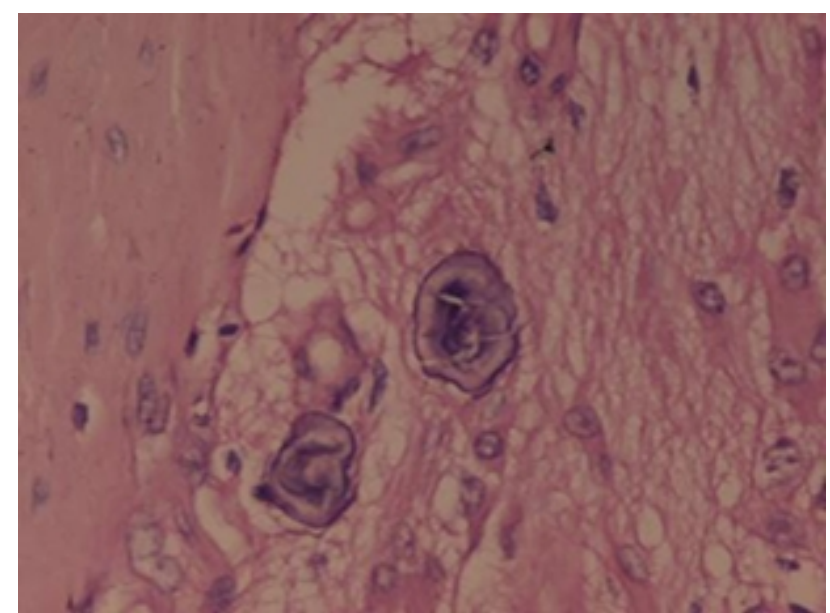

FIG. 3: Photomicrograph shows glial tissue with a fibrillary background and interspersed fibrovascular stroma with psammomatous calcifications. Haematoxylin and Eosin $\times 40$

The postoperative recovery was uneventful and the cosmetic result was satisfactory. Follow-ups performed over the last 12 months using brain MRI showed no residual parts or recurrence of the glioma.

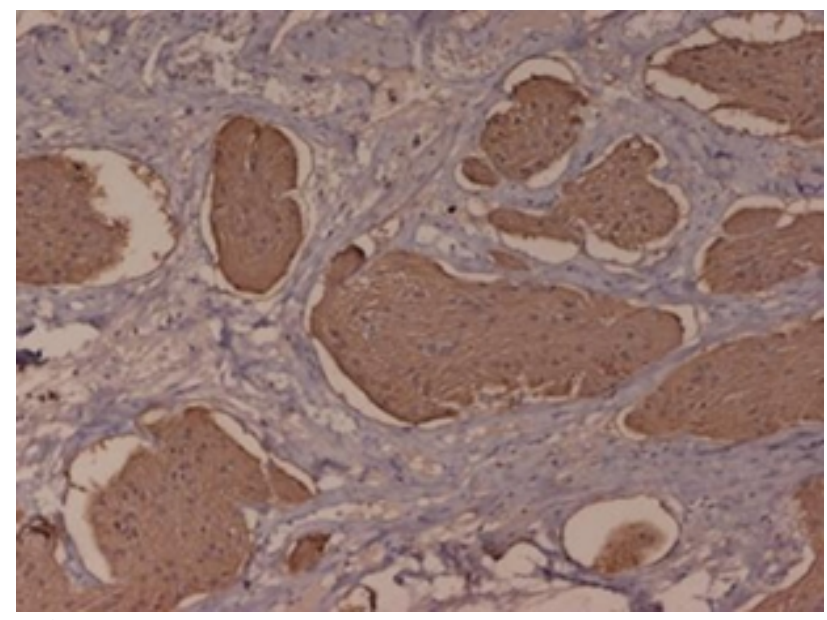

FIG. 4: Photomicrograph shows glial tissue with a positive reaction for S100 protein. S-100 $\times 10$

\section{Discussion}

The term nasal glioma has been used to describe a congenital benign tumor of the nasal region containing neural tissue. Schmidt was the first scientist to describe the comprehensive nature of the nasal glioma in 1900. However, the term he used is a misnomer. ${ }^{3,5}$ Nasal gliomas are not true neoplasms; they originate from ectopic glial tissue left extracranially following abnormal closure of the nasal and frontal bone during embryonic development. ${ }^{1,4,6}$ Therefore, some authors recommend using the term 'glial heterotopia' instead. ${ }^{3}$ Nasal gliomas generally present at birth, rarely in adults, as a mass without associated nasal symptoms. They have a 3:1 male predominance, with no familial or hereditary predisposition 
and no malignant potential., 7 The tumour growth rate is consistent with the patient's body growth. There are intranasal (30\%), extranasal $(60 \%)$, or combined (10\%) types of nasal gliomas. ${ }^{6}$ Our case was of an extranasal glioma.

Most nasal gliomas are diagnosed at birth or during early childhood, with relative peaks of occurrence between 5 and 10 years of age. Although the majority of patients present with signs and symptoms during the first year of life, a later presentation may be due to a specialist's failure to recognize a subclinical lesion in childhood or because of a trauma. ${ }^{2,5}$ Unlike dermoids, they do not necessarily occur in the midline, or attach to sinuses or skin. Gliomas form an uncompressible mass that does not increase in size on the Valsalva testing and does not transilluminate. Extranasal gliomas are usually located at the glabella level, but they may be present laterally. Intranasal gliomas are associated most frequently with the middle turbinate or higher structures, and may mimic nasal polyps. Combined intra/extranasal gliomas have a typical dumbbell shape with a connecting band. Fifteen percents of gliomas are connected with the dura, either through the foramen cecum or through the fonticulus. $\mathrm{Pa}$ tients may present with a unilateral nasal obstruction, unilateral nasal mass, epistaxis, or cerebrospinal rhinorrhea. ${ }^{4,5}$

As gliomas are nonencapsulated accumulations of glial cells situated outside the CNS, the possible theories of development include the following: 1) sequestration of glial tissue of the olfactory bulb entrapped during cribriform plate fusion; 2 ) ectopic neural tissue cells; 3) pinched encephalocele; and 4) inappropriate closure of the anterior neuropore (fonticulus frontalis), with the failure of mesoderm to enter the region, resulting in an inadequate bone formation. ${ }^{7,8}$

Histologically, nasal gliomas are composed of astrocytes and neuroglial cells, embedded in fibrous and vascular connective tissue. ${ }^{5}$ They have no true capsule and mitosis is rarely noted. Multinucleated or gemistocytic astrocytes may be present but it is rare to find neurons. The presence of abundant neurons raises the possibility of an encephalocoele. The glial nature of the cells can be further confirmed by immunohistochemical demonstration of S100 protein and GFAP. These two proteins can identify neurological cells with high specificity, and help to distinguish nasal gliomas from other tumours such as meningiomas and granular cell tumours. ${ }^{5,8}$ In our case, both S100 and GFAP staining were positive. Hence, a definitive diagnosis of nasal glioma was made.

The clinical differential diagnosis includes several various congenital and acquired disorders, which could be manifested by nasal masses. Nasal glioma should be differentiated from several common or important lesions, among which: 1) Nasal dermoids, which are epithelial-lined cavities or sinus tracts with variable numbers of skin appendages, including hair follicles, sebaceous glands, and eccrine glands.
They constitute the most common congenital nasal anomaly. 2) Encephaloceles point to the herniation of neural tissue through defects in the skull. They may contain meninges (meningocele) or brain matter and meninges (encephalomeningocele), or they may communicate with a ventricle (encephalomeningocystocele). Encephaloceles have an etiology similar to that of gliomas. Twenty percents of all encephaloceles occur in the cranium. Of those, $15 \%$ are nasal. 3) Hemangioma, which are the most frequent benign vascular tumors in infancy. ${ }^{9-11}$

The diagnosis is corroborated by CT scan, which shows a bony defect. MR imaging is obligatory in patients with suspected glioma to determine possible intracranial extension of the nasal cavity lesion and the brain. ${ }^{12,13}$

The treatment of choice of nasal gliomas is complete surgical excision. ${ }^{1}$ Gliomas are benign but incomplete excision results in a $4 \%$ to $10 \%$ recurrence rate. The approach depends upon the location and extent of the lesion and levels of expertise available. When facilities are available, intranasal endoscopic surgery is considered most appropriate approach for the removal of intranasal glioma having no intracranial extension as this approach allows precise excision with minimal trauma to the surrounding tissues. ${ }^{9}$ If however intracranial extension is evident, than frontal craniotomy, multidisciplinary team approach may be required in specialized neurosurgical or craniofacial centers to ensure complete and safe excision of the lesions. ${ }^{10}$

\section{Conclusion}

Nasal gliomas are rare congenital anomalies. It is mandatory to rule out intracranial extension by cross-sectional imaging, preferably by MRI before performing any invasive procedure. Conservative surgical excision is the mainstay of the management of an extranasal glioma, as it is slow-growing, rarely recurrent, and has no malignant potential.

\section{Conflict of interest}

The authors declare that they have no conflicts of interest. The authors alone are responsible for the content and writing of the paper.

\section{References}

1. Chang KC, Leu YS. Nasal glioma: A case report. Ear Nose \& Throat J2001; 80: 410-1.

2. Ducic Y. Nasal Gliomas. J Otolaryngol 1999; 28: 285-7.

3. Verney Y, Zanolla G, Teixeira R, Oliveira LC. Midline nasal mass in infancy: a nasal glioma case report. Eur J Pediatr Surg 2001; 11: 324-327. 
4. Hyams VJ, Batsakis JG, Michaels L. Tumors of the Upper Respiratory Tract and Ear. Atlas of Tumor Pathology, 2 ${ }^{\text {nd }}$ series, Fascicle 25, Armed Forces Institute of Pathology, Washington D.C; 1988: 251-7.

5. Cerda-Nicolas M, Sanchez Fernandez de Sevilla C, Lopez-Gines C, Peydro-Olaya A, Llombart- Bosch A. Nasal glioma or nasal heterotopia? Morphological, immunohistochemical and ultrastructural study of two cases. Clin Neuropathol 2002; 21:66-71.

6. Michaels L and Hellquist HB. Neuroectodermal Tumours in Ear, Nose and Throat Histopathology, $2^{\text {nd }}$ ed. Berlin: Springer; 2001: 203-17.

7. Verney Y, Zanolla G, Teixeira R, Oliveira LC. Midline nasal mass in infancy: a nasal glioma case report. Eur J Pediatr Surg 2001; 11: 324-7.
8. Nada V, Dejan V, Dragan D, Ljiljana J. Nasal glioma. Archive of Oncology 2006; 14: 57-9.

9. Hoeger PH, Schaefer H, Ussmueller J, Helmke K. Nasal glioma presenting as capillary haemangioma. Eur J Pediatr 2001; 160: 84-7.

10. Dasgupta NR and Bentz ML. Nasal gliomas: identification and differentiation from hemangiomas. $J$ Craniofac Surg 2003; 14: 736-8.

11. Jartti PH, Jartti AE, Karttunen AI, Paakko EL, Herva RL, Pirila TO. MR of a nasal glioma in a young infant. Acta Radiol 2002; 43:141-3.

12. Yokoi H, Wada R, Ichikawa G. Endoscopic treatment of so-called intranasal glioma. Rhinology 2002; 40: 217-9. 